\title{
Middle lobe syndrome: an extraordinary presentation of endobronchial tuberculosis
}

The authors declare no financial disclosure

\begin{abstract}
Tuberculous infection of the tracheobronchial tree, termed as endobronchial tuberculosis (EBTB), is more common in young adults and females. This clinical entity is poorly understood and the diagnosis is frequently delayed as sputum smears are often negative for acid fast bacilli and the chest radiograph can be normal, resulting in diagnostic confusion. Bronchoscopy continues to play a key role in its diagnosis. Though atelectasis is not uncommon in these patients, EBTB presenting as a middle lobe syndrome (MLS) has rarely been documented. MLS refers to chronic or recurrent collapse of the right middle lobe and has a myriad of causes. The pathogenesis of this entity too is not fully established. We report this exceptional clinical manifestation in a 19-year-old male, who presented for evaluation of respiratory symptoms for 6 months along with constitutional complaints. Imaging suggested the presence of MLS and bronchoscopy established the diagnosis of endobronchial tuberculosis. GeneXpert evaluation of bronchial aspirate detected Mycobacterium tuberculosis. Histopathology confirmed the presence of granulomatous lesions. Subsequently, the cultures of bronchial aspirate and post-bronchoscopy sputum grew M. tuberculosis. Appropriate therapy with anti-tuberculosis drugs resulted in a remarkable symptomatic and radiological improvement. EBTB presenting as a MLS is a distinct rarity.
\end{abstract}

Key words: chest imaging, endobronchial tuberculosis, fibreoptic bronchoscopy, middle lobe syndrome, Mycobacterium tuberculosis

Pneumonol Alergol Pol 2015; 83: 387-391

\section{Introduction}

Tuberculous infection of the tracheobronchial tree is known as endobronchial tuberculosis (EBTB) and in patients with active pulmonary tuberculosis, its frequency ranges from $10-50 \%$ [1-3]. It can occur with or without parenchymal involvement and is more common in young adults and females [3, 4]. EBTB is a distinct form of tuberculosis which continues to remain a diagnostic challenge even in high tuberculous prevalent countries. The chest X-ray in EBTB can be normal and diagnostic confirmation can only be achieved bronchoscopically. This variant of tuberculosis is highly infectious and can consequently prove to be dangerous since it is a potential source of infection in the community. Diagnostic confusion can occur as it can simulate major pulmonary disorders like bronchial asthma and lung cancer. Bronchial stenosis is a well-known and dreaded complication of EBTB which can result in atelectasis and obstructive pneumonia. Till date, the pathogenesis of EBTB is ill understood and the clinical course can be variable [3, 4].

Chronic or recurrent collapse of the right middle lobe was termed as middle lobe syndrome (MLS) by Graham et al. [5] in 1948. However, it was Brock [6] who first described recurrent collapse of right middle lobe due to enlarged tuberculous lymph nodes and is still widely known as "Brock's syndrome". MLS continues to fascinate clinicians as it is a distinct but an uncommon

Address for correspondence: Ashok Shah, Department of Respiratory Medicine, Vallabhbhai Patel Chest Institute, University of Delhi, Delhi 110007 , India, e-mail: ashokshah99@yahoo.com

DOI: 10.5603/PiAP.2015.0062

Received: 28.05 .2015

Copyright (C) 2015 PTChP

ISSN 0867-7077 


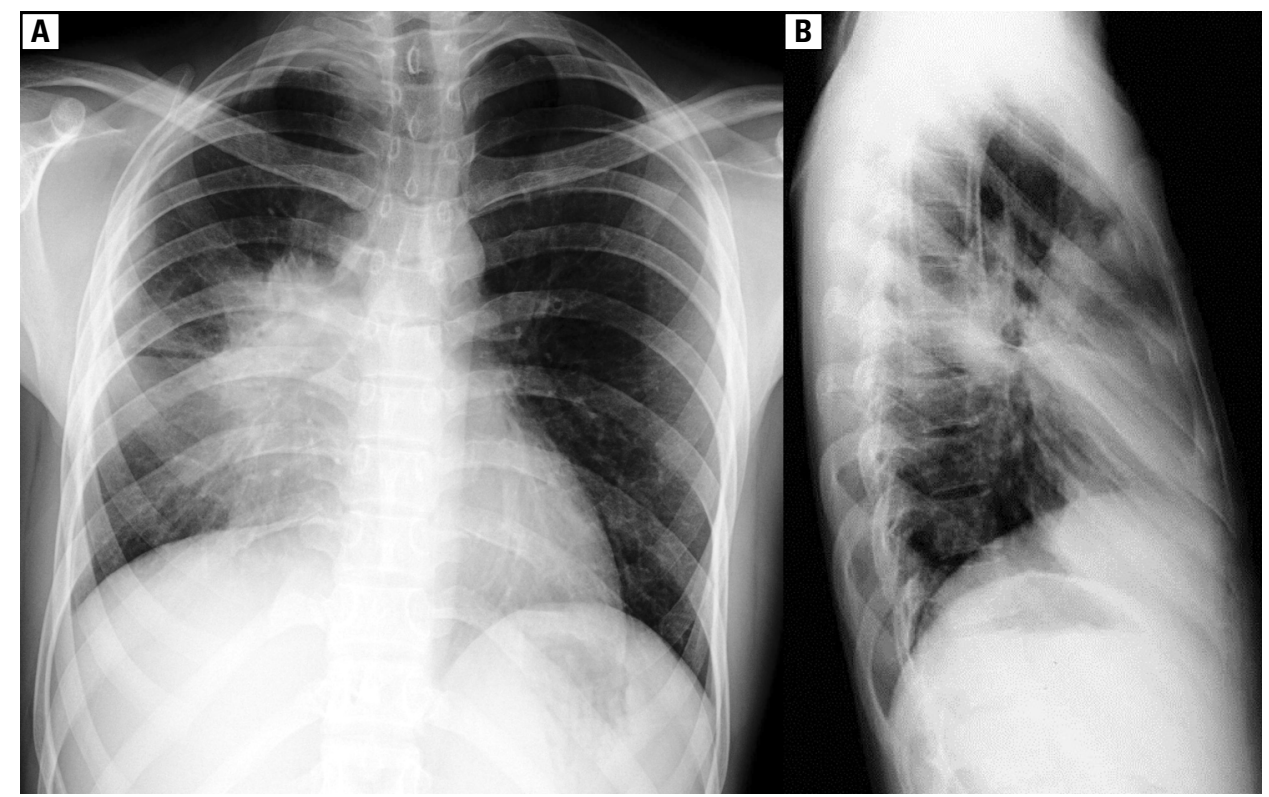

Figure 1. Chest radiograph (posteroanterior view) showed an ill-defined opacity in the right middle zone abutting the right cardiac border with loss of cardiac silhouette (A); chest radiograph (right lateral view) showed a wedge shaped density extending from the hilum anteriorly and inferiorly along with loss of volume suggestive of MLS (B)

clinical presentation which is yet to be fully appreciated. A wide array of respiratory disorders is known to cause MLS and like EBTB, it occurs more commonly in females.

Although MLS due to tuberculosis is a well -defined clinical entity [7, 8], MLS as a presentation of EBTB is a distinct rarity. A search of the literature revealed only 2 reports documenting 25 patients with EBTB who presented with MLS [9, 10]. One documented 22 patients from Korea [9] and the other reported three from Turkey [10] suggesting that this phenomenon is singularly uncommon. This is supported by the fact that in younger patients with EBTB, trachea and the main bronchi are the most frequently affected sites rather than the middle lobe [9]. Dearth of the literature on the subject prompted this report of a 19-year-old man who presented with MLS due to EBTB.

\section{Case report}

A 19-year-old HIV-negative male, a never smoker, was referred to our Institute for evaluation of dry cough, right sided chest pain and evening rise of temperature for 6 months along with loss of appetite and weight for 1 month. He also had exertional dyspnoea for 3 months.

General physical examination revealed a young boy in no acute respiratory distress. Pallor, clubbing, cyanosis or peripheral lymphadenopathy were not present. Examination of the respiratory system did not reveal any abnormality.

Haemogram, renal and hepatic functions were within normal limits. The chest radiograph showed an ill-defined opacity in the right middle zone abutting the right cardiac border with loss of cardiac silhouette (Fig. 1A). The right lateral view showed a wedge shaped density extending from the hilum anteriorly and inferiorly along with loss of volume suggestive of MLS (Fig. 1B). High resolution contrast enhanced computed tomography (CECT) of the thorax revealed mediastinal lymphadenopathy compressing the right middle lobe bronchus (Fig. 2A: mediastinal window) accompanied by a trapezoidal opacity with its base towards the hilum and contiguous with the right cardiac border confirming the presence of MLS (Fig. 2B: mediastinal window). Mantoux test with 1 TU elicited an induration of $20 \mathrm{~mm}$ after 48 hours. In spite of nebulization with hypertonic saline, the patient was unable to produce sputum for examination. Fibreoptic bronchoscopy visualised a narrowed right middle lobe bronchus with swollen, hyperaemic mucosa and thick white cheesy necrotic secretions emanating from the right middle lobe (Fig. 3A, B). Bronchial aspirate and the post-bronchoscopy sputum were negative for AFB but GeneXpert detected Mycobacterium tuberculosis without rifampicin resistance. Transbronchial biopsy showed granulomatous inflammation consisting of epithelioid cells and multinucleated giant 


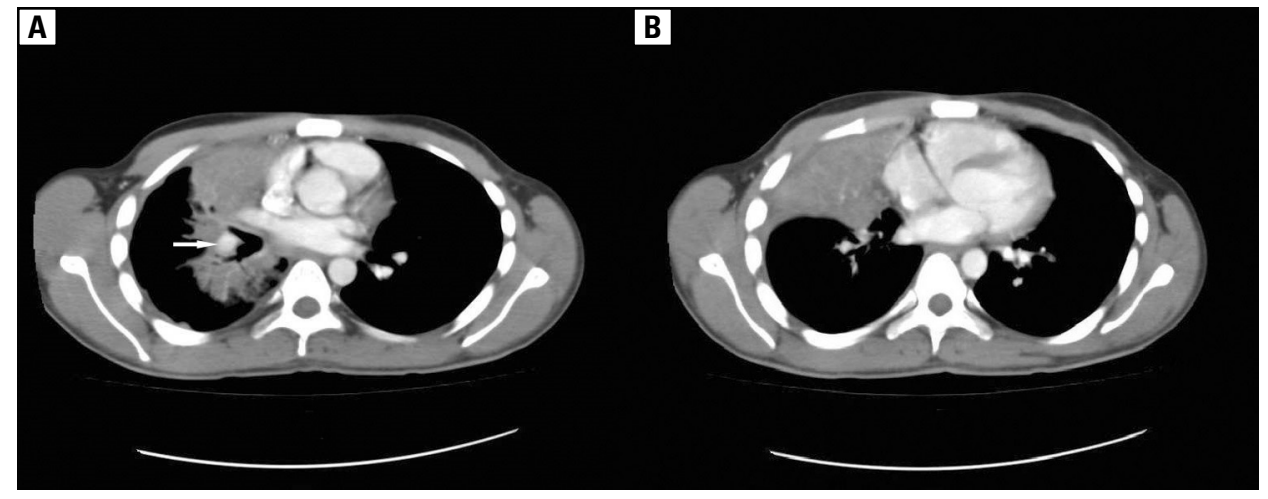

Figure 2. A - CECT of the thorax (mediastinal window) showed mediastinal lymphadenopathy compressing the right middle lobe bronchus (Arrow); B - CECT of the thorax (mediastinal window) showed a trapezoidal opacity with its base towards the hilum and contiguous with the right cardiac border confirming the presence of MLS. Air bronchograms were not seen in the opacity

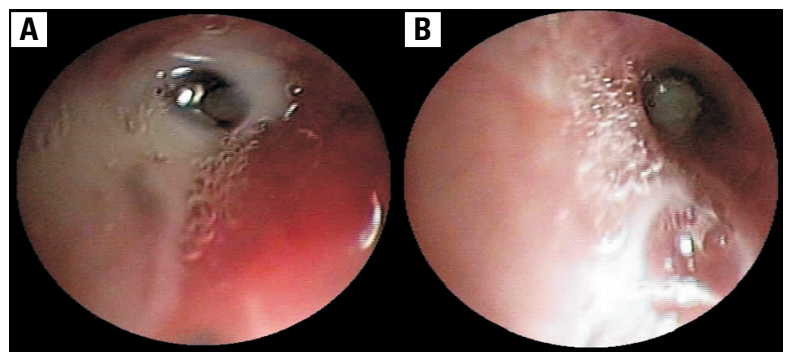

Figures 3. A, B. Fibreoptic bronchoscopy showed a narrowed right middle lobe bronchus with swollen, hyperaemic mucosa and thick white cheesy necrotic secretions emanating from the right middle lobe

cells with numerous lymphocytes consistent with the diagnosis of tuberculosis. Subsequently, Mycobacterium tuberculosis was isolated from bronchial aspirate as well as post-bronchoscopy sputum.

A diagnosis of endobronchial tuberculosis presenting as a MLS was made on the basis of the following: 1) on bronchoscopy, the middle lobe bronchial mucosa was oedematous, hyperaemic with cheesy whitish tenacious secretions emanating from the narrowed middle lobe (Fig. 3) suggestive of actively caseating type of lesions [11], 2) positive GeneXpert, 3) transbronchial biopsy confirming granulomatous inflammation, 4) subsequent isolation of Mycobacterium tuberculosis from bronchial aspirate and post-bronchoscopy sputum and 5) characteristic radiological presentation suggestive of right middle lobe syndrome.

The patient was initiated on standard first line antituberculous therapy consisting of isoniazid (300 mg), rifampicin (450 mg), pyrazinamide (1500 mg), ethambutol $(800 \mathrm{mg}$ ) once daily. He experienced remarkable symptomatic relief within a fortnight and marked radiological clearance was observed after two months.

\section{Discussion}

MLS is usually divided into obstructive and non-obstructive types. Obstruction can be due to both intraluminal as well as extraluminal obstruction and is thought to be a feature characteristic of MLS [12]. The non-obstructive form is caused by inflammation with benign inflammatory lesions being the most common causes of MLS and is seen in $62 \%$. Primary tumours account for $24 \%$ while tuberculosis is responsible for $8-10 \%$ [13]. In an earlier study from Poland, MLS was observed in $25 \%$ of the elderly females with tuberculosis [14]. Recently, tuberculosis was documented in 2/20 (10\%) children with MLS from Turkey [15] and in 8/40 (20\%) adults with MLS from Iran [16]. Other less common causes include allergic bronchopulmonary aspergillosis [17] and bronchial anthracofibrosis [8].

It is hypothesised that middle lobe has a greater susceptibility to collapse as its bronchus is rather narrow where it originates. The long length as well as the angular take-off of this bronchus makes it liable to be compressed due to enlarged lymph nodes. In addition, poor collateral ventilation as a consequence of the anatomical separation of the middle lobe from the right upper and lower lobes by fissures along with inadequate clearance of the impacted mucous can also contribute to the propensity to collapse in isolation [12]. In children with tuberculosis, it is often difficult to distinguish whether MLS is due to bronchial obstruction or due to the sequelae of nodal compression [13]. In the pre-chemotherapeutic era, of the 66 children with collapse due to tuberculosis, the right middle lobe was affected in 23 (34\%) [18]. In our patient, enlarged tuberculous lymph nodes around the middle lobe bronchus 
caused extrinsic compression resulting in a MLS. Moreover, cheesy white tenacious secretions seen in the middle lobe bronchus of our patient may have caused intraluminal obstruction.

Imaging is crucial to the diagnosis of MLS with the chest radiograph showing obliteration of the right cardiac border (silhouette sign) due to the proximity of the medial segment of the middle lobe with the right atrium. However, the collapse is better visualised on the lateral chest radiograph as a triangle of increased density extending from hilum anteriorly and inferiorly with its apex at the hilum and the base lying peripherally at the pleura. The presence of trapezoidal or broad triangular opacity with base towards the hilum, contiguous with the right cardiac border on CT thorax confirms the diagnosis of MLS. In addition, the CT can also evaluate bronchial patency, lymph node enlargement responsible for MLS, as was seen in our patient. Endobronchial as well as parenchymal abnormalities can also be visualised [12].

There can be a considerable diagnostic delay in patients with EBTB as chest radiograph can be normal while bronchoscopy, which is confirmatory of the diagnosis, is usually not done in patients with pulmonary tuberculosis. Furthermore, sputum smears for AFB are positive in only $17 \%$ of patients with EBTB but the diagnostic yield can be dramatically increased to $79 \%$ when bronchoscopic specimens are combined [4]. In our patient too, the initial smears of bronchial aspirate and post-bronchoscopy sputum were negative for AFB but culture of both these samples subsequently yielded $M$. tuberculosis.

Radiologically, endobronchial tuberculosis has a varied presentation but can also be normal in $10-20 \%$ of the cases $[19,20]$. A retrospective study of 55 patients with endobronchial tuberculosis documented patchy alveolar infiltration, atelectasis, cavitary lesions and hilar enlargement. Although atelectasis was detected on CT in 16 patients, none of them had a MLS [20]. A recent study from Korea documented the occurrence of EBTB in 233/429 (54.3\%) patients with pulmonary tuberculosis. Of the 233 patients with EBTB, atelectasis was observed in $42(18 \%)$ but MLS was not documented in any of the 429 patients [3]. However, the occurrence of MLS in EBTB has been documented in 22 patients from Korea [9] and an additional three from Turkey [10].

Bronchoscopy is pivotal to the diagnosis of EBTB and endobronchial lesions have been categorised as actively caseating, oedematous -hyperaemic, fibrostenotic, tumorous, granular, ulcerative and non-specific bronchitic [21]. Oedematous type was the dominant lesion seen on bronchoscopy in those presenting as MLS $(15 / 22,68.2 \%)$ [9]. In contrast, the most common lesion detected on bronchoscopy in 233 patients with EBTB without MLS was the actively caseating type $(114 / 233,48.9 \%)$ [3]. In our patient, we found that the middle lobe bronchus was oedematous and hyperaemic and the presence of cheesy whitish secretions suggested actively caseating lesion.

Bronchial stenosis is a dreaded but not uncommon complication of EBTB [9]. The authors state that in their 22 patients of EBTB with MLS, in spite of adequate and complete treatment, persistent abnormality on chest X-ray was found in more than $50 \%$ patients [9]. Oedematous type of EBTB usually results in a persistent abnormality on chest X-ray while actively caseating type frequently clears up completely with minimal fibrotic change after adequate treatment [11]. In our patient too, there was radiological resolution of the MLS.

In conclusion, the pathogenesis of both EBTB as well as MLS is not fully understood. Bronchoscopy is central to the diagnosis of EBTB as the sputum smears are frequently negative and chest radiograph can be normal. MLS continues to intrigue with its distinct radiological picture and the wide spectrum of diseases that can present with this clinical entity. Although EBTB can present as a MLS, this phenomenon is a distinct rarity and a high index of suspicion would be required to establish the diagnosis. Early recognition and appropriate therapy can possibly prevent complications of this disease.

\section{Conflict of interest}

The authors declare no conflict of interest.

\section{References:}

1. Chung HS, Han SK, Sim YS et al. Balloon dilatation of bronchial stenosis in endobronchial tuberculosis. Tuberculosis Respir Dis 1991; 38: 236-244.

2. Um SW, Yoon YS, Lee SM et al. Predictors of persistent airway stenosis in patients with endobronchial tuberculosis. Int J Tuberc Lung Dis 2008; 12: 57-62.

3. Jung SS, Park HS, Kim JO, Kim SY. Incidence and clinical predictors of endobronchial tuberculosis in patients with pulmonary tuberculosis. Respirology 2015; 20: 488-495. doi: 10.1111/resp.12474.4.

4. Lee P. Endobronchial tuberculosis. Indian J Tuberc 2015; 62: 7-12. doi: 10.1016/j.ijtb.2015.02.002.

5. Graham EA, Burford TH, Mayer JH. Middle lobe syndrome. Postgrad Med 1948; 4: 29-34.

6. Brock RC, Cann RJ, Dickinson JR. Tuberculous mediastinal lymphadenitis in childhood; secondary effects on the lungs. Guy’s Hospital Resp 1937; 87: 295. 
7. Gupta PP, Gupta KB, Agarwal D. Middle lobe syndrome due to tuberculous etiology: a series of 12 cases. Indian J Tuberc 2006; 53: 104-108.

8. Kala J, Sahay S, Shah A. Bronchoanthracofibrosis and tuberculosis presenting as a middle lobe syndrome. Prim Care Respir J 2008; 17: 51-55. doi: 10.3132/pcrj.2008.00003.

9. Kim HC, Kim HS, Lee SJ et al. Endobronchial tuberculosis presenting as right middle lobe syndrome: clinical characteristics and bronchoscopic findings in 22 cases. Yonsei Med J 2008; 49: 615-619. doi: 10.3349/ymj.2008.49.4.615.

10. Sahin F, Yildiz P. Characteristics of endobronchial tuberculosis patients with negative sputum acid-fast bacillus. J Thorac Dis 2013; 5: 764-770. doi: 10.3978/j.issn.20721439.2013.12.15.

11. Chung HS, Lee JH. Bronchoscopic assessment of the evolution of endobronchial tuberculosis. Chest 2000; 117: $385-392$.

12. Gudbjartsson T, Gudmundsson G. Middle lobe syndrome: a review of clinicopathological features, diagnosis and treatment. Respiration 2012; 84: 80-86. doi: $10.1159 / 000336238$.

13. Wagner RB, Johnston MR. Middle lobe syndrome. Ann Thorac Surg 1983; 35: 679-686.
14. Sedlaczek AM. Analysis of the clinical course of pulmonary tuberculosis in women over 70 years of age. Pneumonol Pol 1990; 58: 210-214.

15. Sehitogullari A, Sayir F, Cobanoglu U, Bilici S. Surgical treatment of right middle lobe syndrome in children. Ann Thorac Med 2012; 7: 8-11. doi: 10.4103/1817-1737.91554.

16. Pejhan S, Salehi F, Niusha S, Farzanegan B, Sheikhy K. Ten years' experience in surgical treatment of right middle lobe syndrome. Ann Thorac Cardiovasc Surg 2015; 21: 354-358. doi: 10.5761/atcs.oa.14-00273.

17. Shah A, Behera S, Panjabi C. Middle lobe syndrome: a rare presentation of allergic bronchopulmonary aspergillosis. Eur Ann Allergy Clin Immunol 2014; 46: 147-151.

18. Richards WF. Bronchial obstructions in primary pulmonary tuberculosis. Proc R Soc Med 1944; 37: 589-592.

19. Ip MS, So SY, Lam WK et al. Endobronchial tuberculosis revisited. Chest 1986; 89: 727-730.

20. Yanardag H, Tetikkurt C, Tetikkurt S, Demirci S, Karayel T. Computed tomography and bronchoscopy in endobronchial tuberculosis. Can Respir J 2003; 10: 445-448.

21. Chung HS, Lee JH, Han SK, et al. Classification of endobronchial tuberculosis by the bronchoscopic features. Tuberc Respir Dis 1991; 38: 108-115. 